\title{
Methylphenidate Normalizes Fronto-Striatal Underactivation During Interference Inhibition in Medication-Naïve Boys with Attention-Deficit Hyperactivity Disorder
}

\author{
Katya Rubia*,', Rozmin Halari', Ana Cubillo', Anna B Smith, Abdul-Majeed Mohammad', Michael Brammer² \\ and Eric Taylor'
}

'Department of Child Psychiatry, Institute of Psychiatry, King's College London, London, UK: ${ }^{2}$ Department of Neuroimaging, Institute of Psychiatry, King's College London, London, UK

\begin{abstract}
Youth with attention deficit hyperactivity disorder (ADHD) have deficits in interference inhibition, which can be improved with the indirect catecholamine agonist methylphenidate (MPH). Functional magnetic resonance imaging was used to investigate the effects of a single dose of MPH on brain activation during interference inhibition in medication-naïve ADHD boys. Medication-naïve boys with ADHD were scanned twice, in a randomized, double-blind design, under either a single clinical dose of MPH or placebo, while performing a Simon task that measures interference inhibition and controls for the oddball effect of low-frequency appearance of incongruent trials. Brain activation was compared within patients under either drug condition. To test for potential normalization effects of $\mathrm{MPH}$, brain activation in ADHD patients under either drug condition was compared with that of healthy age-matched comparison boys. During incongruent trials compared with congruent-oddball trials, boys with ADHD under placebo relative to controls showed reduced brain activation in typical areas of interference inhibition, including right inferior prefrontal cortex, left striatum and thalamus, mid-cingulate/supplementary motor area, and left superior temporal lobe. MPH relative to placebo upregulated brain activation in right inferior prefrontal and premotor cortices. Under the MPH condition, patients relative to controls no longer showed the reduced activation in right inferior prefrontal and striato-thalamic regions. Effect size comparison, furthermore, showed that these normalization effects were significant. MPH significantly normalized the fronto-striatal underfunctioning in ADHD patients relative to controls during interference inhibition, but did not affect medial frontal or temporal dysfunction. MPH therefore appears to have a region-specific upregulation effect on fronto-striatal activation.

Neuropsychopharmacology (20 II) 36, I575-1586; doi: I 0. I038/npp.20II.30; published online 30 March 201 I
\end{abstract}

Keywords: attention deficit hyperactivity disorder; methylphenidate; stop task; performance monitoring; error processing; motor response inhibition

\section{INTRODUCTION}

Childhood attention deficit hyperactivity disorder (ADHD) is defined by age-inappropriate inattention, impulsiveness, and hyperactivity (DSM IV) (American Psychiatric Association, 1994). ADHD has consistently been associated with neuropsychological deficits in tasks of motor and interference inhibition (Rubia et al, 2010a, 2011; Willcutt et al, 2005). This has been underpinned by neuroimaging

*Correspondence: Professor K Rubia, Department of Child Psychiatry, SGDP PO46, Institute of Psychiatry, King's College University London, 16 De Crespigny Park, De Crespigny Park, London SE5 8AF, UK, Tel: + 44207848 0463, Fax: + 44207848 0866,

E-mail: katya.rubia@kcl.ac.uk

Received 7 October 2010; revised I February 2011; accepted 2 February 2011 evidence for reduced activation of inhibition-associated inferior prefrontal and caudate regions (Konrad et al, 2006; Rubia et al, 1999, 2005, 2008, 2010a), as well as more generic regions of cognitive control and attention in cingulate and parieto-temporal cortices during these and related tasks (Rubia et al, 1999, 2005, 2008, 2010a, 2011; Vaidya et al, 2005). Psychostimulants, including methylphenidate (MPH) and amphetamines, are the most effective, first-choice treatment for ADHD, improving symptoms in $70 \%$ of patients (Arnsten, 2006a; Wilens, 2008). MPH is a catecholamine reuptake inhibitor with stronger dopamine (DA) upregulating effects in striatal regions and both DA and noradrenaline upregulating effects in cortical areas (Arnsten, 2006a). There is consistent evidence that the behavioral and cognitive features of ADHD are mediated, at least in part, by catecholamine dysfunction, 
given that adults with ADHD have abnormal striatal DA transporter (DAT) levels and reduced DA availability in the basal ganglia (Krause, 2008; Volkow et al, 2006, 2007a, b). Relatively little, however, is known on the effects of MPH on brain function in ADHD. MPH has been shown to improve performance on motor and interference inhibition tasks (DeVito et al, 2009; Langleben et al, 2006; Tannock et al, 1989). Relatively few functional magnetic resonance imaging (fMRI) studies, however, have investigated the acute effects of MPH on neural networks of inhibition functions. Vaidya et al (1998) found that the single dose of MPH upregulated defined regions of interest of caudate, anterior cingulate, and frontal brain regions in previously medicated children with ADHD during motor response inhibition. A recent study found no effect of a single dose MPH administration on the neural networks of interference inhibition in medication-responsive patients with ADHD, but on anterior and posterior cingulate activation during the control condition, which was interpreted as enhancement of the default-mode network suppression (Peterson et al, 2009). A study in adults with ADHD testing for 6 weeks of chronic effects of MPH on brain activation during a multi-source interference inhibition task found an enhancement effect on anterior cingulate as well as dorsolateral prefrontal, striato-thalamic, and parietal activation (Bush et al, 2008). All these studies were conducted in previously medicated patients with ADHD. Longitudinal studies, however, suggest that long-term medication may have an effect on both brain structure (Shaw et al, 2009) and function (Konrad et al, 2007).

To overcome these limitations, we aimed to investigate the effect of $\mathrm{MPH}$ on neural processes of interference inhibition in medication-naïve boys with ADHD. Imaging studies of interference inhibition tasks are typically confounded by the mismatch in frequency between low frequent interference inhibition and high frequent congruent trials, co-measuring the attentional oddball effect to less frequent trials. To avoid this confound, we used our fMRI paradigm of the Simon task that incorporates an oddball task to control for the attention allocation effect (Rubia et al, 2011; Smith et al, 2006). In healthy adolescents, task performance activates bilateral dorsolateral and inferior prefrontal, basal ganglia, thalamus, anterior cingulate, and temporal brain regions (Christakou et al, 2009a; Rubia et al, 2006). Children with ADHD relative to controls have shown reduced activation in fronto-striatal, medial frontal/anterior cingulate, and temporo-parietal regions during this (Rubia et $a l, 2009 \mathrm{c}, 2011)$ and similar interference inhibition tasks (Konrad et al, 2006; Vaidya et al, 2005).

We hence conducted a randomized, double-blind, placebo-controlled pharmacological fMRI experiment to test for the effects of a single acute clinical dose of MPH on brain activation in 12 medication-naive boys with ADHD during interference inhibition. Furthermore, brain activation in ADHD patients at baseline and after MPH was compared with that of a healthy age-matched control group, to test for potential amelioration or normalization effects of MPH on brain dysfunctions during the placebo condition. During tasks of sustained attention and time estimation, we previously observed normalization of fronto-striatal and parietal brain activation in medication-naïve patients with ADHD (Rubia et al, 2009a, b). We therefore hypothesized that during interference inhibition, MPH would upregulate and normalize areas of underactivation that are typically observed in ADHD patients relative to controls during this task in inferior and medial frontal/anterior cingulate and striatal brain regions.

\section{METHODS}

\section{Subjects}

Twelve male medication-naïve, right-handed boys aged 10-15 years who met clinical diagnostic criteria for the combined (inattentive-hyperactive) subtype of ADHD (DSM-IV), with a mean age of 13 years $(S D=1)$, were recruited through clinics. One additional patient was recruited but not included in the study owing to movement artifacts and non-completion of the second scan. Clinical diagnosis of ADHD was established through interviews with an experienced child psychiatrist (A-MM) using the standardized Maudsley diagnostic interview to check the presence or absence of each of the criteria and applying the numerical cutoffs for numbers of symptoms in each domain as set out by DSM-IV (Goldberg and Murray, 2002). The Maudsley diagnostic interview contains items on symptoms of ADHD, autism, conduct, bipolar, affective and anxiety, and other pervasive developmental disorders. Items were then combined into diagnoses following the rules of the ICD-10 (Research Diagnostic Criteria), except that for ADHD the criteria of the DSM-IV (TR) were followed. Exclusion criteria were lifetime co-morbidity with any other psychiatric disorder, except for conduct/oppositional defiant disorder (present in one patient), as well as learning disability and specific reading disorder (as assessed by the full developmental and educational history of the child, taken from parent/carer, by school information, and by excluding subjects with a Raven's IQ lower than the 5th percentile), neurological abnormalities, epilepsy, drug or substance abuse, and previous exposure to stimulant medication. Patients with ADHD had to also score above cutoff for hyperactive-inattentive symptoms on the Strengths and Difficulties Questionnaire (SDQ) for parents (Goodman and Scott, 1999). Patients were scanned twice, in a randomized, counter-balanced manner, 1 week apart, $1 \mathrm{~h}$ after either $0.3 \mathrm{mg} / \mathrm{kg}$ of $\mathrm{MPH}$ administration or placebo (vitamin C, $100 \mathrm{mg}$ ).

Thirteen male right-handed boys in the age range of $11-$ 16 years (mean age $=13, \mathrm{SD}=1$ ) were recruited through advertisements in the same geographic areas of South London to ensure similar socioeconomic status. They scored below the cutoff for behavioral problems in the SDQ, and had no history of psychiatric disorder.

All participants were above the 5 th percentile on the Raven progressive matrices performance IQ (Raven, 1960) (IQ mean estimate: controls $=102, \mathrm{SD}=15 ; \mathrm{ADHD}=90$, $\mathrm{SD}=9)$ and paid $£ 30$ for participation. Parental and child informed consent/assent and approval from the local Ethical Committee was obtained.

Univariate ANOVAs showed no group differences between boys with $\mathrm{ADHD}$ and controls for age $(\mathrm{F}(1.25)=1.7, p=0.2)$, but there was a significant difference in IQ $(\mathrm{F}(1.25)=5.5$, $p<0.023)$. Consequently, all between-group performance and imaging data analyses were covaried for IQ. 


\section{fMRI Paradigm: Simon Task}

Subjects practiced the Simon task once before scanning. The 6 min fMRI adaptation of the Simon task involves a stimulus-response incompatibility effect and measures interference inhibition and selective attention. To control for the attentional oddball effect of low-frequency appearance of incongruent trials, the task contains an oddball condition (Rubia et al, 2006, 2009c, 2011; Smith et al, 2006).

Subjects have to press a left/right button depending on whether an arrow stimulus of $300 \mathrm{~ms}$ duration points either to the left or right side of the screen. The mean ITI was $1.8 \mathrm{~s}$, but jittered between 1.6 and $2 \mathrm{~s}$ for optimal statistical efficiency of fast event-related fMRI data analysis (Dale, 1999). In congruent trials (160 trials), the arrow pointing left (right) appears on the left (right) side of the screen. In $12 \%$ of trials (24 trials), arrows appear on the opposite side of where they point and subjects have to inhibit responding according to the interfering, predominant spatial information while continuing to respond to the iconic information (arrow direction). To control for the attentional oddball effect of the low-frequency appearance of the incongruent trials, slightly slanted 'oddball', but congruent stimuli appeared in another $12 \%$ of trials (24 trials), to which subjects have to respond to as to the congruent stimuli.

The event-related analysis compares successfully performed incongruent with successfully performed oddball trials to measure the neural correlates of interference inhibition, controlling for the attentional oddball effect (incongruent-oddball trials).

\section{fMRI Image Acquisition}

Gradient-echo echoplanar MRI data were acquired on a GE Signa $1.5 \mathrm{~T}$ Horizon LX System (General; Electric, Milwaukee, WI) at the Maudsley Hospital (London, UK). A quadrature birdcage head coil was used for RF transmission and reception. In each of the 16 non-contiguous planes parallel to the anterior-posterior commissural, 208 $\mathrm{T}_{2}^{*}$-weighted $\mathrm{MR}$ images depicting BOLD (Blood Oxygen Level Dependent) contrast covering the whole brain were acquired with $\mathrm{TE}=40 \mathrm{~ms}, \mathrm{TR}=1.8 \mathrm{~s}$, flip angle $=90^{\circ}$, inplane resolution $=3.1 \mathrm{~mm}$, slice thickness $=7 \mathrm{~mm}$, and slice skip $=0.7 \mathrm{~mm}$, providing complete brain coverage.

\section{fMRI Image Analysis}

The method of fMRI analysis used (XBAM, http:// www.brainmap.co.uk) (Brammer et al, 1997) makes no normality assumptions, which are usually violated in fMRI data, but instead uses median statistics to control outlier effects and permutation, rather than normal theory-based inference. Furthermore, the most common test statistic is computed by standardizing for individual difference in residual noise before embarking on second-level, multisubject testing using robust permutation-based methods. This allows a mixed-effects approach to analysis - an approach that has recently been recommended following a detailed analysis of the validity and impact of normal theory-based inference in fMRI in large number of subjects (Thirion et al, 2007).

\section{Individual Analyses}

fMRI data were realigned to minimize motion-related artifacts (Bullmore et al, 1999) and smoothed using a Gaussian filter (full-width half-maximum, $7.2 \mathrm{~mm}$ ). Timeseries analysis of individual subject activation was performed using XBAM, with a wavelet-based re-sampling method described previously (Bullmore et al, 2001). Briefly, we first convolved each experimental condition with two Poisson model functions (delays of 4 and $8 \mathrm{~s}$ ). Only correct trials were included in the analyses (ie correct incongruent/ Simon and correct oddball trials, both contrasted with an implicit baseline, that is, congruent trials). We then calculated the weighted sum of these two convolutions that gave the best fit (least squares) to the time series at each voxel.

A goodness-of-fit statistic (the SSQ-ratio) was then computed at each voxel consisting of the ratio of the sum of squares of deviations from the mean intensity value due to the model (fitted time series) divided by the sum of squares due to the residuals (original time series minus model time series). The appropriate null distribution for assessing significance of any given SSQ-ratio was established using the wavelet-based data re-sampling method (Bullmore et al, 2001) and applying the model-fitting process to the re-sampled data. This process was repeated 20 times at each voxel and the data combined over all voxels, resulting in 20 null parametric maps of SSQ-ratio for each subject, which were combined to give the overall null distribution of SSQ-ratio. The same permutation strategy was applied at each voxel to preserve spatial correlation structure in the data. Activated voxels, at a $<1$ level of type I error, were identified through the appropriate critical value of the SSQ-ratio from the null distribution. Individual SSQ-ratio maps were then transformed into standard space, first by rigid body transformation of the fMRI data into a high-resolution inversion recovery image of the same subject, and then by affine transformation onto a Talairach template (Talairach and Tournoux, 1988).

\section{Group Analyses}

A group activation map was then produced for the experimental condition of incongruent-oddball trials by calculating the median observed SSQ-ratio over all subjects at each voxel in standard space and testing them against the null distribution of median SSQ-ratios computed from the identically transformed wavelet re-sampled data (Brammer et al, 1997). The voxel-level threshold was first set to 0.05 to give maximum sensitivity and to avoid type II errors. Next, a cluster-level threshold was computed for the resulting three-dimensional voxel clusters such that the final expected number of type I error clusters was $<1$ per whole brain. The necessary combination of voxel and cluster-level thresholds was not assumed from theory, but rather was determined by direct permutation for each data set, giving excellent type II error control (Bullmore et al, 1999). Cluster mass rather than a cluster extent threshold was used, to minimize discrimination against possible small, strongly responding foci of activation (Bullmore et al, 1999). For the group activation analyses, less than one false-positive 
activation locus was expected for $p<0.05$ at voxel level and $p<0.05$ at cluster level.

\section{Within- and Between-Group Comparisons}

For the within-group comparisons of the effect of MPH $v s$ placebo on brain activation, one-way repeated measures ANOVA analyses were conducted using randomizationbased tests for voxel- or cluster-wise differences as described in detail (Brammer et al, 1997; Bullmore et al, 2001). This was done for the contrast condition Simon-oddball. Less than one false-activated cluster was expected at $p<0.05$ for voxel comparison and $p<0.01$ for cluster comparison for these tests.

To test for hypothesized amelioration/normalization effects of MPH on brain activation compared with healthy controls, two ANCOVAs with IQ estimate as covariate were conducted: (1) between controls and boys with ADHD under the placebo condition and (2) between controls and boys with ADHD under the MPH condition. Less than one false-activated cluster was expected at $p<0.05$ for voxel comparison and $p<0.01$ for cluster comparison.

\section{RESULTS}

\section{Performance Data}

Within-patient comparison between MPH and placebo. Repeated measures ANOVAs within ADHD patients showed no significant difference between placebo or medication on overall reaction times (RTs) (d.f. $=1,11 ; \mathrm{F}=1, p<0.3$ ), overall error rates (d.f. $=1.11 ; \mathrm{F}=0.001, p<0.9$ ), or on the difference in RT between incongruent and congruent trials (Simon RT effect) (d.f. $=1.11 ; \mathrm{F}=0.05, p<0.8$ ) or the Simon accuracy effect (differences in accuracy between incongruent and congruent trials) (d.f. $=1.11 ; \mathrm{F}=0.2$, $p<0.7$ ) (see Table 1).

Comparison between ADHD patients under placebo compared with controls. Univariate ANCOVAs showed that ADHD patients under placebo were slower than controls in RTs (d.f. $=1.24 ; \mathrm{F}=7, p<0.017$ ), but did not differ in errors (d.f. $=1.24 ; \mathrm{F}=0.8, p<0.4)$. Repeated measures ANCOVA for the Simon RT time and Simon accuracy effect showed that there was no overall Simon effect for RTs (d.f. $=1.22 ; \mathrm{F}=0.6, p=0.4)$ and a trend-level significance for a Simon accuracy effect, which was due to larger error numbers in the incongruent compared to congruent trials (d.f. $=1.22 ; \mathrm{F}=2, p<0.1$ ). However, no significant interaction was observed between group and Simon RT effect (d.f. $=1.22 ; \mathrm{F}=0.2, p=0.7$ ) or between group and Simon accuracy effect (d.f. $=1.22 ; \mathrm{F}=2, p=0.2$ ) (see Table 1).

Comparison between ADHD patients under MPH compared with controls. Univariate ANCOVAs showed that ADHD patients under MPH were still slower than controls in RT (d.f. $=1.24 ; \mathrm{F}=5, p<0.035$ ), but did not differ in errors (d.f. $=1.24 ; \mathrm{F}=1, \quad p<0.2$ ). Repeated measures ANOVA for the Simon RT and Simon accuracy effect showed that there was no overall Simon effect for RT (d.f. $=1.22 ; \quad F=0.8, \quad p=0.4$ ), but a significant Simon accuracy effect, due to larger error numbers in the incongruent compared to congruent trials $(\mathrm{F}=2$, $p<0.042)$. However, no significant interaction was observed between group and Simon RT effect (RT: d.f. $=1.22 ; \mathrm{F}=0.4$, $p=0.5$ ) or between group and Simon accuracy effect (RT: d.f. $=1.22 ; \mathrm{F}=2, p=0.2$ ).

\section{Brain Activation}

Movement. There were no significant differences in the extent of three-dimensional motion parameters between controls and ADHD boys under placebo for $x, y, z$ rotation (d.f. $=3.21 ; \mathrm{F}=2, p<0.14$ ) or $x, y, z$ translation (d.f. $=3.21$; $\mathrm{F}=1.3, p<0.3)$ nor between controls and ADHD boys under MPH in $x, y, z$ rotation (d.f. $=3.21 ; \mathrm{F}=1.7, p<0.2$ ) or $x, y, z$ translation (d.f. $=3,21 ; \mathrm{F}=2, p<0.15$ ). Nor were there any differences in motion parameters for the drug comparison within patients for $x, y, z$ rotation (d.f. $=3,20$; $\mathrm{F}=0.3, p<0.8$.) or $x, y, z$ translation (d.f. $=3,20 ; \mathrm{F}=1$, $p<0.4)$.

One subject was excluded owing to high head movement of over $2 \mathrm{~mm}$ in the $x, y$, and $z$ rotation and translation (see Methods).

Within-group brain activations. Controls activated relatively large clusters in right inferior prefrontal cortex, reaching deep into insula, basal ganglia, and thalamus, in left insula, basal ganglia, and thalamus, in left and right dorsolateral prefrontal cortex, medial frontal cortex, including anterior cingulate and supplementary motor area (SMA), in left and right inferior parietal lobes and in right superior temporal lobe.

Activation in ADHD patients under placebo was in right inferior prefrontal cortex, reaching into insula, caudate and putamen, in left inferior prefrontal gyrus, in ventromedial frontal cortex, including anterior cingulate and SMA, in thalamus, bilateral inferior parietal lobe, and in left middle temporal gyrus.

Table I Main Variables of the Simon Task by Group

\begin{tabular}{lccc}
\hline Performance measure & Healthy controls, $\mathbf{N}=\mathbf{I 3}$ & ADHD placebo, $\mathbf{N}=\mathbf{I} \mathbf{2}$ & ADHD MPH, N=I \\
\hline MRT congruent (ms) & $430(72)$ & $54 \mid(\mid 24)$ & $504(124)$ \\
MRT incongruent (ms) & $537(89)$ & $620(122)$ & $579(128)$ \\
Error rate congruent (\%) & $2(2)$ & $3(2)$ & $2(3)$ \\
Error rate incongruent (\%) & $23(14)$ & $19(9)$ & $19(15)$
\end{tabular}

Abbreviation: MRT, mean reaction time. 
Table 2 Within-Group ANOVA Differences in Brain Activation in Boys with ADHD between Placebo and Methylphenidate for the Simon Task

\begin{tabular}{|c|c|c|c|c|}
\hline Brain region & BA & Tal. coordinates $(x ; y ; z)$ & No. of voxels & Cluster $p$-value \\
\hline \multicolumn{5}{|l|}{ Methylphenidate > placebo } \\
\hline L cerebellum/fusiform/middle/inferior temporal & $37 / 21 / 19$ & $-11 ;-52 ;-13$ & 163 & 0.001 \\
\hline \multicolumn{5}{|l|}{ Placebo > methylphenidate } \\
\hline
\end{tabular}

Abbreviation: BA, Brodman area.

Activation in ADHD patients under MPH was in right inferior prefrontal cortex, reaching into insula, caudate and putamen, and in medial frontal cortex, including anterior cingulate and SMA (see Supplementary Figure 1).

ANOVA within-patient comparisons in brain activation between the placebo and the MPH conditions. MPH contrasted with placebo elicited enhanced activation in two large clusters: one comprised right inferior prefrontal and premotor cortex reaching into superior temporal and inferior parietal lobes, and the other cluster comprised left cerebellum and middle and inferior temporal lobes (see Table 2, Figure 1). The placebo condition elicited no enhanced activation over MPH.

Comparison between controls and ADHD patients under either placebo or $M P H$. Relative to controls, ADHD patients under the placebo condition showed underactivation in right inferior prefrontal cortex reaching into inferior parietal lobe, in left ventromedial frontal cortex, the basal ganglia, and thalamus, in a large cluster comprising right SMA, anterior and posterior cingulate gyri, and in left superior and middle temporal/occipital cortex (Table 3, Figure 2).

Under the $\mathrm{MPH}$ condition, ADHD patients showed reduced activation in the same clusters, with exception of two previous difference clusters of activation, the one in the left ventromedial frontal cortex, basal ganglia, and thalamus, and the other one in right inferior prefrontal cortex. Between-group differences in these two clusters were no longer observed (Figure 2, Table 3).

To establish whether the group differences between control boys and ADHD boys under the two drug comparisons in the inferior frontal and basal ganglia clusters were significantly different, we directly compared the effect sizes of the group differences in activation resulting from the two comparisons (controls compared with ADHD under placebo, and controls compared with ADHD under MPH) (Matthews and Altman, 1996). When comparing two effect sizes, the $z$-test can evaluate the likelihood of whether they are significantly different. The difference between the two effect sizes (es) can be considered a normalized variable, where the standard error of the difference (se) is a combination of the standard errors of the two comparisons. Based on this, the probability of a type I error can be calculated using the following formula: $p(\alpha)=\left(\mathrm{es}_{1}-\mathrm{es}_{2}\right) / \operatorname{sqrt}\left(\mathrm{se}_{1}^{2}+\mathrm{se}_{2}^{2}\right) \quad$ (Matthews and Altman, 1996).

The effect size comparison showed that effect sizes for the group differences in the inferior prefrontal activation cluster for the two comparisons were significantly different (Statistical power of BOLD response: control, 0.0142; ADHD placebo, -0.0153 ; ADHD MPH, 0.087; $z=-1.6 ; p<0.05)$ as were the effect sizes for the group differences for the two comparisons in the basal ganglia activation cluster (Statistical power of BOLD response: control, 0.0042; ADHD placebo, -0.0236; ADHD MPH, 0.024; $z=0.5 ; p<0.04$ ).

Given that the brain activation differences were based on the higher-level contrast between Simon and Oddball conditions, we elucidated further these activation differences by extracting BOLD responses in these between-group difference clusters for the contrast of Simon-Oddball condition as well as for the lower-level contrasts of Simon-Congruent and Oddball-Congruent trials in both groups. As can be observed in Figure $3 a$ for the ANCOVA difference clusters for the comparison between control boys and ADHD boys under placebo, the group differences in all clusters were arisen because control boys recruited these brain regions to a greater extent for the Simon-Congruent condition than for the Oddball-Congruent condition, whereas in patients with $\mathrm{ADHD}$, these brain regions were more activated in the Oddball-Congruent condition than in the Simon-Congruent condition. In other words, in healthy boys these brain regions shifted towards greater activation from the Oddball to the Simon condition, whereas in ADHD patients, these brain regions showed a greater response during the Oddball condition than the Simon condition.

For the comparison between control boys and ADHD patients under $\mathrm{MPH}$, the same patterns were observed for the three brain clusters that remained different between groups. In all three activation clusters, brain activation for the Simon-Congruent condition was higher in controls than for Oddball-Congruent condition, whereas ADHD patients showed a more pronounced activation in these regions during the Oddball-Congruent condition than for the Simon-Congruent condition (see Figure $3 \mathrm{~b}$ ).

In addition, we wanted to test whether the intensity of the brain underactivation in ADHD patients relative to controls during the placebo condition was associated with symptom severity. For this purpose, statistical measures of BOLD response for each ADHD participant was extracted in each of the significant clusters of between-group activation differences during the placebo condition and then 


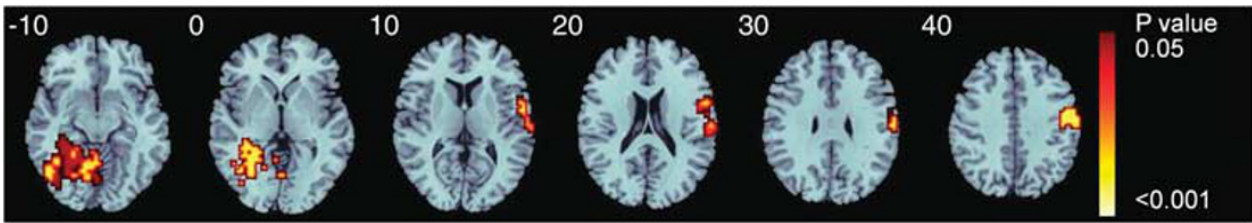

Figure I Increased brain activation with the single dose of methylphenidate compared with placebo in boys with attention deficit hyperactivity disorder (ADHD) during interference inhibition. Axial slices for within-group analysis of variance (ANOVA) results comparing methylphenidate and placebo for the contrast of incongruent-oddball trials at family-wise error-corrected cluster-level contrast of $p<0.0$ I. Methylphenidate compared with placebo enhanced activation in right inferior/premotor cortex and in a cluster comprising left cerebellum and middle and inferior temporal lobes. No brain regions were enhanced under placebo compared with methylphenidate. Talairach z-coordinates are indicated for slice distance (in mm) from the intercommissural line. The right side of the figure corresponds to the right side of the brain. The color bars indicate $p$-values with lighter colors reflecting more significant $p$-values.

Table 3 Between-Group ANCOVA Differences in Brain Activation between Control Boys and Boys with ADHD Under Either the Placebo or the Methylphenidate Condition for the Contrast of Simon vs Oddball Condition

\begin{tabular}{|c|c|c|c|c|}
\hline Brain region & BA & Tal. coordinates $(x ; y ; z)$ & No. of voxels & Cluster $p$-value \\
\hline \multicolumn{5}{|l|}{ Controls > ADHD under placebo } \\
\hline $\mathrm{R}$ inferior frontal/inferior parietal ${ }^{\mathrm{a}}$ & $45 / 9 / 40$ & $18 ;-33 ; 59$ & 182 & 0.007 \\
\hline R SMA/anterior/posterior cingulate/superior parietal & $6 / 24 / 7$ & $4 ;-11 ; 37$ & 282 & 0.004 \\
\hline L superior/middle temporal/occipital & $22 / 39$ & $-43 ;-37 ; 20$ & 69 & 0.003 \\
\hline L SMA/anterior cingulate/precuneus & $24 / 32 / 6 / 7$ & $-22 ;-11 ; 48$ & 297 & 0.006 \\
\hline L middle temporal/occipital & 19/37 & $-36 ;-67 ;-13$ & 53 & 0.001 \\
\hline L superior temporal/inferior parietal/precuneus & 22/40/7 & $-36 ;-30 ; 20$ & 49 & 0.007 \\
\hline
\end{tabular}

Abbreviations: BA, Brodman area; L, left; N voxels, number of voxels; R, right; SMA, supplementary motor area; Tal. coordinates, Talairach coordinates.

$P$-value for ANCOVAs at family-wise error-corrected cluster-level contrast of $p<0.01$. Boys with ADHD under placebo had no increased activation compared with controls for either condition.

${ }^{a}$ No differences were observed between boys with ADHD under methylphenidate and healthy control boys in these two clusters.

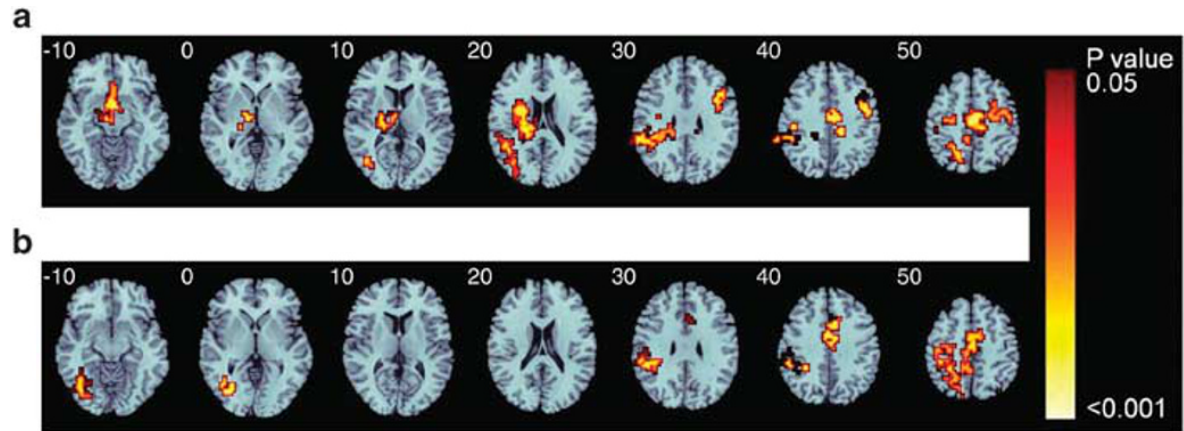

Figure 2 (a) Axial slices showing significantly reduced activation in boys with attention deficit hyperactivity disorder (ADHD) under placebo compared with healthy comparison boys at family-wise error-corrected cluster-level contrast (analysis of covariance (ANCOVA)) of $p<0.0$ I for the contrast of incongruent-oddball trials in the Simon task. No increased activation was observed in ADHD patients compared with healthy controls. (b) Under the methylphenidate condition, brain activation differences between groups were no longer observed in the clusters in right inferior/dorsolateral prefrontal cortex (DLPFC) and the left caudate/thalamus/ventromedial frontal lobe. Talairach z-coordinates are indicated for slice distance (in mm) from the intercommissural line. The right side of the figure corresponds to the right side of the brain. The color bars indicate $p$-values with lighter colors reflecting more significant $p$-values.

correlated with inattentive-hyperactive symptoms on the SDQ. We observed a negative correlation within the ADHD group between the activation in the cluster in the basal ganglia and inattentive-hyperactive symptoms on the SDQ $(r=-0.7 ; p<0.016$, two-tailed); no other correlations were observed.

\section{DISCUSSION}

ADHD patients did not significantly differ from controls in task performance. MPH relative to placebo had no significant ameliorating effect on task performance within patients or on the differences relative to healthy controls. 
a Right inferior frontal
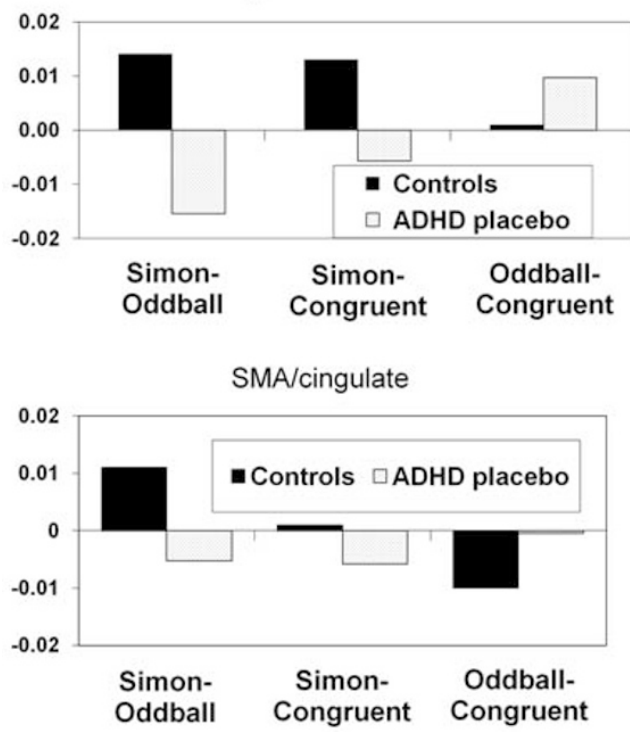

b SMA/cingulate

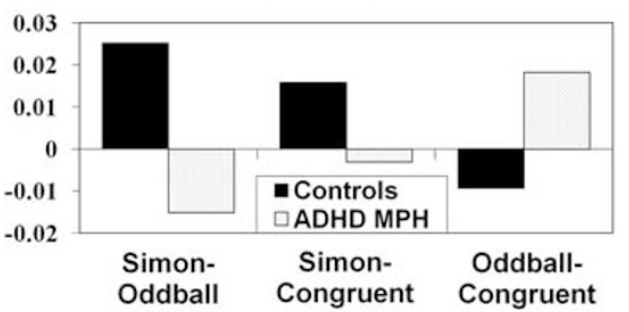

Left vmPFC/basal ganglia
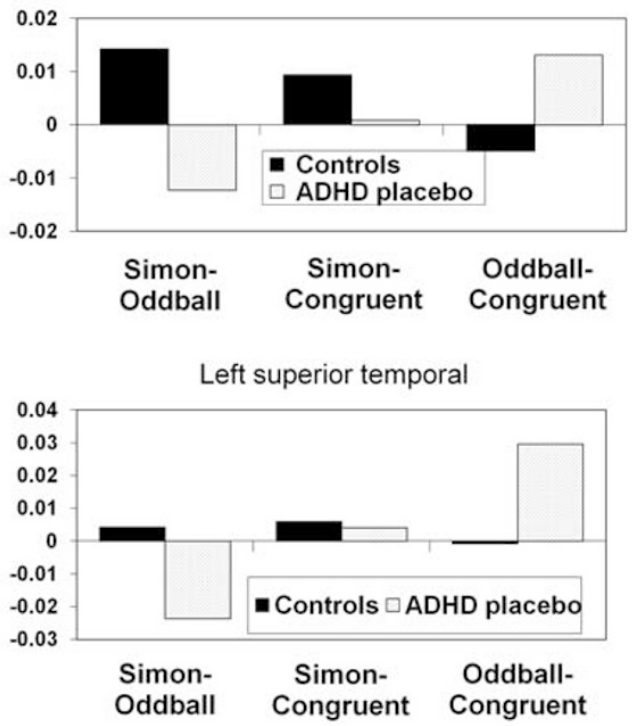

Left superior temporal

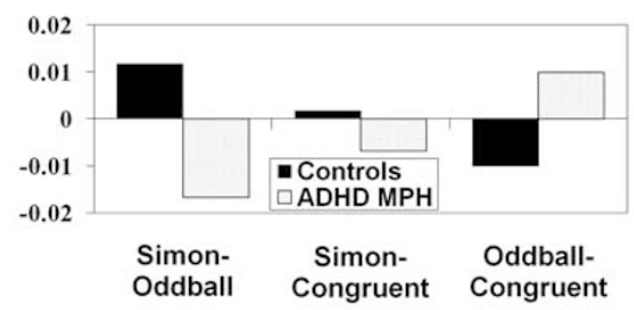

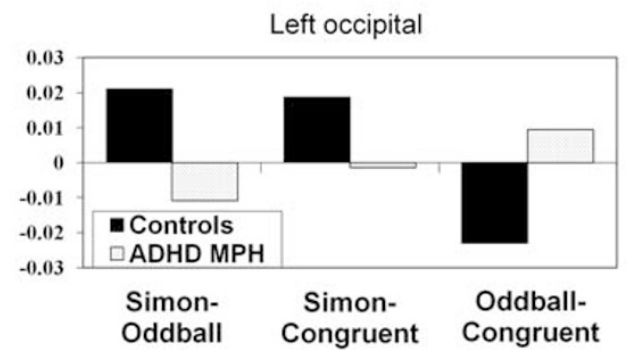

Figure 3 Standardized BOLD (Blood Oxygen Level Dependent) responses in areas of analysis of covariance (ANCOVA) group differences for (a) comparison between healthy control boys and attention deficit hyperactivity disorder (ADHD) boys under the placebo condition and (b) comparison between healthy control boys and ADHD boys under the methylphenidate condition. The color bars indicate $p$-values with lighter colors reflecting higher $p$-values.

Nevertheless, MPH relative to placebo had significant upregulation and normalization effects on brain activation. MPH elicited enhanced activation within ADHD patients in right inferior prefrontal and premotor cortices as well as in left cerebellum and inferior and middle temporal lobes. When patients under placebo were compared with controls, they showed reduced activation in right inferior prefrontal cortex reaching into inferior parietal lobe, in left basal ganglia and thalamus, in ventromedial and dorsomedial prefrontal, and in temporal regions. The underactivation in basal ganglia, thalamus, and ventromedial frontal lobe, furthermore, was negatively associated with symptom severity. No brain regions were increased in activation in patients relative to controls. All activation deficit clusters relative to controls remained when patients were under $\mathrm{MPH}$, with the exception of the underactivation clusters in right inferior prefrontal cortex, and the symptom-severityassociated cluster in left basal ganglia/thalamus and ventromedial prefrontal cortex. Furthermore, the regionspecific normalization effects were significant, given that the effect sizes of the activation differences in these two activation clusters were significantly larger for the comparison between controls and ADHD under placebo than for the comparison between controls and ADHD under MPH. The findings suggest that a single clinical dose of MPH has region-specific normalization effects on abnormal brain activation in ADHD patients in inferior and ventromedial fronto-striatal networks during interference inhibition.

Right inferior prefrontal cortex as well as the caudate and thalamus form part of a fronto-striatal network of motor and interference inhibition in adults and children (Aron and Poldrack, 2006; Li et al, 2008; Christakou et al, 2009a; 
Rubia, 2007c; Rubia et al, 2003, 2006). Furthermore, the underactivation in these brain regions for ADHD patients was specific to the interference condition, as the group differences in these regions seemed to arise from the fact that control boys showed more activation in these areas for the Simon condition, whereas ADHD patients showed more activation in these regions during the Oddball condition. Inferior prefrontal underactivation in the context of tasks of cognitive control is one of the most consistent findings in fMRI studies in patients with ADHD (Dickstein et al, 2006; Durston et al, 2003; Konrad et al, 2006; Rubia et al, 1999, 2005; Vaidya et al, 1998, 2005; for a review see Rubia (2011)). Inferior prefrontal cortex, as well as caudate and thalamus, have, furthermore, been found to be dysfunctional in ADHD children during other, more generic attention functions such as performance monitoring (Pliszka et al, 2006; Rubia et al, 2010a), selective, sustained, and flexible attention tasks (Rubia et al, 2007b, 2009b, c, d, 2010a, 2011; Smith et al, 2006). Inferior prefrontal dysfunction, furthermore, appears to be a disorder-specific neurofunctional deficit compared to patients with conduct (Rubia et al, 2008, 2009c, d, 2010b) and obsessive-compulsive disorder (Rubia et al, 2010a), whereas striatal underactivation appears to be disorder specific relative to OCD patients (Rubia et al, 2010a,2011; for a review see Rubia (2010)). In these data, furthermore, the activation in the basal ganglia cluster correlated with symptom severity, so that the ADHD patients with more severe inattention and hyperactivity symptoms had more reduced activation. MPH therefore appears to modulate an important neuro-functional biomarker of ADHD, a dysfunction in fronto-striatal neural networks that mediate cognitive control.

The findings of upregulation of activation in inferior prefrontal cortex and the basal ganglia under a single dose of MPH extend previous findings of upregulation of these brain regions in the context of other tasks. Thus, lateral prefrontal and caudate activation has previously been shown to be upregulated, but not normalized in ADHD patients with a single clinical dose of MPH during tasks of motor response inhibition (Vaidya et al, 1998). Furthermore, our findings of a significant specific normalization effect on the activation in left basal ganglia, but not in temporal lobes, is in line with the study of Shafritz et al (2004), who also found a region-specific upregulation and normalization effect of MPH on left striatal underactivation, but not on the underactivation of middle temporal lobe. The finding of upregulation and normalization of right inferior prefrontal/premotor cortex with $\mathrm{MPH}$ is in line with strikingly similar findings of upregulation and normalization of underfunctioning relative to controls in this region in the same subjects with the same clinical dose of MPH during a sustained attention task (Rubia et al, 2009b). The upregulation effects in right prefrontal, premotor, and left thalamic brain regions under a single dose of MPH is also in line with evidence for longer-term, chronic upregulation effects. Chronic doses of MPH over 6 weeks lead to a significant upregulation of these areas in adults with ADHD during a different interference inhibition task (Bush et al, 2008). The findings of upregulation of frontostriatal brain activation during interference inhibition in ADHD boys in this study, however, are not in line with a recent study of Peterson et al (2009), who found that MPH had no effect on fronto-striatal activation during a Stroop interference inhibition task, but enhanced activation in the control condition of the task in the ventral anterior cingulate and posterior cingulate, which was interpreted as suppression of default-mode activity.

Also, in this study, we did not observe a normalization effect of MPH on the dysfunction in medial frontal activation. Like in this study, the SMA and anterior cingulate have previously been found to be underactivated in ADHD patients during interference inhibition tasks (Bush et al, 1999; Rubia et al, 2011). Previous studies, however, unlike this one, found a modulation of either chronic (Bush et al, 2008) or single doses of MPH (Vaidya et al, 1998) on this structure during motor and interference inhibition, or on the control condition of the task (Peterson et al, 2009). Differences in findings may be due to the differences in task design or differences in medication history, as patients were not medication naïve in these previous studies.

We observed, however, normalization in a more ventromedial frontal location. Upregulation and normalization of abnormal activation in this region has been observed in the same patient group during a time discrimination task (Rubia et al, 2009a). Ventromedial frontal cortex is thought to be important for holding information in representational memory (Schoenbaum et al, 2006) and has been associated with selective attention and decision making (Christakou et al, 2009b).

Posterior thalamic underactivation clusters in patients relative to controls under placebo were also upregulated and normalized with $\mathrm{MPH}$. Posterior thalamic brain regions have been associated both with inhibitory control (Aron and Poldrack, 2006; Li et al, 2008), as well as attention to salient stimuli such as oddball, novel, or incongruent targets (Rubia et al, 2007c, 2009b, c; Stevens et al, 2007; Tamm et al, 2006). The significant normalization effects in inferior and ventromedial frontal, striatal, and thalamic brain regions therefore suggest that MPH appears to normalize activation of all parts of a fronto-striato-thalamic cognitive control network (Rubia et al, 2007c). Furthermore, this network cluster was significantly associated with symptom severity in ADHD patients. This suggests that MPH had a normalization effect on brain deficits that are associated with symptom severity, which may be the mechanism of action that underlies behavioral improvement.

The inferior frontal underactivation cluster that was normalized with the single MPH dose in patients, furthermore, reached into inferior parietal lobe in more superior slices. To our knowledge, normalization of inferior parietal activation with $\mathrm{MPH}$ has only recently been observed in ADHD patients, in the context of sustained attention (Rubia et al, 2009b) and another interference inhibition task (Bush et al, 2008).

$\mathrm{MPH}$ prevents the reuptake of catecholamines from the synaptic cleft by blocking DAT and norepinephrine transporter (NET) (Volkow et al, 1995, 1997). In vitro studies in animals show that MPH has high affinity for the DAT, lower affinity for the NET, and minimum affinity for the serotonin transporter (Bymaster et al, 2002; Gatley et al, 1996). In human kidney cells, MPH has shown to have greater affinity for NET than DAT (Eshleman et al, 1999). Positron emission tomography (PET) studies show that $\mathrm{MPH}$ in healthy adults blocks $60-70 \%$ of striatal DAT in a 
dose-dependent manner and significantly increases levels of extracellular DA in the striatum (Schiffer et al, 2006; Volkow et al, 1997, 2002a, b, 2007a), as well as in frontal, thalamic, and temporal brain regions (Montgomery et al, 2007). The upregulating effects on the caudate activation were therefore likely mediated by effects on the dopaminergic system. In frontal regions, however, studies in rats and mice have shown that MPH upregulates noradrenaline to the same or greater extent than DA (Balcioglu et al, 2009; Berridge et al, 2006). This is thought to be mediated by reuptake inhibition of NET, as NET in frontal regions clear up both DA and noradrenaline, given that there are few DATs in these areas (Moron et al, 2002; Arnsten and Dudley, 2005; Arnsten, 2006b; Berridge et al, 2006; Bymaster et al, 2002; Staller and Faraone, 2007). The effects of MPH on the inferior prefrontal activation could therefore have been associated with both DA and noradrenaline upregulation effects (Arnsten, 2006a). Likewise, the effect on thalamic upregulation may have been mediated by blockage of NETs, as these are densely distributed in the thalamus (Hannestad et al, 2010). Furthermore, a recent PET study showed that MPH at clinically relevant doses significantly occupies $70-80 \%$ of NETs in NET-rich regions, including cortical and thalamic areas, which is larger than the percentage of blockage that has previously been observed on DAT occupancy (Volkow et al, 1998). As opposed to the significantly high blockage of DAT in striatal regions, however, MPH had little effect on NET in the basal ganglia (Hannestad et al, 2010). The upregulating effects on frontal and thalamic activation, therefore, may have been mediated by enhanced DA and noradrenaline neurotransmission caused by NET blockage, whereas basal ganglia upregulation effects were more likely caused by DAT-mediated effects on DA neurotransmission.

Patients compared with controls showed no performance deficits. Evidence for performance deficits in tasks of interference inhibition is controversial in the ADHD literature (Mullane et al, 2009; Rubia et al, 2007a; van Mourik et al, 2005). The negative findings may also be due to the relatively low statistical power for neuropsychological data and the use of an older adolescent age group compared with the childhood age groups previously shown to have performance deficits. The finding of no significant effects of the clinical dose of MPH on performance on the Simon task is in line with previous negative findings of an effect of $\mathrm{MPH}$ in the related Stroop interference inhibition task (Solanto et al, 2009). The findings of brain dysfunctions in patients relative to controls and their upregulation and normalization in boys with ADHD under the clinical dose of $\mathrm{MPH}$ despite no observable performance changes show that brain activation is more sensitive than performance to detect both abnormalities and pharmacological effects. We have previously shown that adolescents with ADHD show marked brain dysfunctions despite no task impairment in this and similar inhibition tasks (Rubia et al, 1999, 2005, 2009c) and brain activation has consistently been shown to be more sensitive than behavior to show pharmacological effects of MPH in ADHD patients (Bush et al, 2008; Konrad et al, 2006; Peterson et al, 2009; Rubia et al, 2009a b; Shafritz et al, 2004).

A limitation of the study is the relatively small sample size. Minimum numbers of 15-20 participants have been suggested for fMRI studies (Thirion et al, 2007). Repeated measures designs, however, are statistically more powerful than independent data sets, which makes the within-subject ANOVA more robust. It cannot be excluded, however, that with larger sample sizes upregulation or normalization effects of MPH could be found for other brain regions. The findings of region-specific normalization effects of MPH on the activation in inferior frontal lobes and the basal ganglia, therefore, need to be considered with caution until replicated in larger datasets.

Another limitation of the study is that patients were tested twice, whereas controls were only scanned once, for ethical and financial reasons. Practice effects, however, were overcome by the counterbalanced design.

Also, this experimental study investigated the effects of one single clinical dose of MPH on brain activation in medication-niive boys with ADHD. Effects of a single acute dose of $\mathrm{MPH}$ are not comparable to long-term MPH treatment effects, where medication is typically titrated and given over longer periods of time. Studies of acute dosage only provide a unique probe of brief changes in catecholamine modulation that can provide insights into the effects of these brief changes on underlying brain function. The findings of this study can therefore not be transferred to elucidate underlying mechanisms of longterm clinical treatment and are hence limited in their applicability to clinical reality.

Furthermore, only male youth were included in the study to increase sample homogeneity. ADHD is more prevalent in boys (Merikangas et $a l, 2010$ ) and gender differences exist in clinical manifestation, cognitive deficits, and brain dysfunctions (Gershon, 2002; Mahone and Wodka, 2008; Valera et al, 2010). The findings may therefore not generalize to the female youth population.

The task design did not include an absolute rest condition, so that the active task condition (Simon condition) was contrasted with lower-level baseline conditions (ie, oddball; congruent trials). We found that the activation differences were due to stronger activation in controls relative to ADHD patients during the active task (incongruent trials) relative to the lower-level baseline conditions. The contrast of the active task condition with an absolute baseline, such as a rest condition, could have provided additional information that might have further clarified the interaction findings. ADHD children, however, are known to differ from controls in their brain activation during the resting state (Konrad and Eickhoff, 2010) and a resting condition may therefore not necessarily be more disambiguating than a lower-level baseline condition.

In conclusion, to our knowledge, this is the first study to show that a single clinical dose of $\mathrm{MPH}$ in medication-naïve youth with ADHD has a region-specific effect of significantly normalizing symptom-associated fronto-striatal underfunctioning during interference inhibition.

\section{ACKNOWLEDGEMENTS}

This research was funded by grants from the Wellcome Trust, UK to KR (053272/Z/98/Z/JRS/JP/JAT) and the Medical Research Council (G9900839), UK to ET. RH was 
supported by a grant from the PPP Healthcare UK to KR $(1206 / 1140)$.

\section{DISCLOSURE}

KR has received funding from Eli Lilly for another research project and speakers honoraria from Eli Lilly and Medice. The other authors declare no conflict of interest.

\section{REFERENCES}

American Psychiatric Association (1994). Diagnostic and Statistical Manual of Mental Disorders, 4th edn. American Psychiatric Association: Washington, DC.

Arnsten AF (2006a). Fundamentals of attention-deficit/hyperactivity disorder: circuits and pathways. J Clin Psychiatry 67(Suppl 8): 7-12.

Arnsten AF, Dudley AG (2005). Methylphenidate improves prefrontal cortical cognitive function through alpha2 adrenoceptor and dopamine D1 receptor actions: relevance to therapeutic effects in attention deficit hyperactivity disorder. Behav Brain Funct 1: 2.

Arnsten AFT (2006b). Stimulants: therapeutic actions in ADHD. Neuropsychopharmacology 31: 2376-2383.

Aron AR, Poldrack RA (2006). Cortical and subcortical contributions to stop signal response inhibition: role of the subthalamic nucleus. J Neurosci 26: 2424-2433.

Balcioglu A, Ren JQ, McCarthy D, Spencer TJ, Biederman J, Bhide PG (2009). Plasma and brain concentrations of oral therapeutic doses of methylphenidate and their impact on brain monoamine content in mice. Neuropharmacology 57: 687-693.

Berridge CW, Devilbiss DM, Andrzejewski ME, Arnsten AF, Kelley AE, Schmeichel B et al (2006). Methylphenidate preferentially increases catecholamine neurotransmission within the prefrontal cortex at low doses that enhance cognitive function. Biol Psychiatry 60: 1111-1120.

Brammer MJ, Bullmore ET, Simmons A, Williams SC, Grasby PM, Howard RJ et al (1997). Generic brain activation mapping in functional magnetic resonance imaging: a nonparametric approach. Magnet Reson Imag 15: 763-770.

Bullmore E, Long C, Suckling J, Fadili J, Calvert G, Zelaya F et al (2001). Colored noise and computational inference in neurophysiological (fMRI) time series analysis: resampling methods in time and wavelet domains. Hum Brain Mapp 12: 61-78.

Bullmore ET, Suckling J, Overmeyer S, Rabe-Hesketh S, Taylor E, Brammer MJ (1999). Global, voxel, and cluster tests, by theory and permutation, for a difference between two groups of structural MR images of the brain. IEEE Trans Med Imag 18: 32-42.

Bush G, Frazier JA, Rauch SL, Seidman LJ, Whalen PJ, Jenike MA et al (1999). Anterior cingulate cortex dysfunction in attentiondeficit/hyperactivity disorder revealed by fMRI and the counting Stroop. Biol Psychiatry 45: 1542-1552.

Bush G, Spencer TJ, Holmes J, Shin LM, Valera EM, Seidman LJ et al (2008). Functional magnetic resonance imaging of methylphenidate and placebo in attention-deficit/hyperactivity disorder during the multi-source interference task. Arch Gen Psychiatry 65: 102-114.

Bymaster FP, Katner JS, Nelson DL, Hemrick-Luecke SK, Threlkeld PG, Heiligenstein JH et al (2002). Atomoxetine increases extracellular levels of norepinephrine and dopamine in prefrontal cortex of rat: a potential mechanism for efficacy in attention deficit/hyperactivity disorder. Neuropsychopharmacology 27: 699-711.

Christakou A, Brammer M, Giampietro V, Rubia K (2009b). Right ventromedial and dorsolateral prefrontal cortices mediate adaptive decisions under ambiguity by integrating choice utility and outcome evaluation. J Neurosci 29: 11020-11028.

Christakou A, Halari R, Smith AB, Ifkovits E, Brammer M, Rubia K (2009a). Sex-dependent age modulation of frontostriatal and temporo-parietal activation during cognitive control. NeuroImage 48: 223-236.

Dale AM (1999). Optimal design for event-related fMRI. Hum Brain Mapp 8: 109-114.

DeVito EE, Blackwell AD, Clark L, Kent L, Dezsery AM, Turner DC et al (2009). Methylphenidate improves response inhibition but not reflection-impulsivity in children with attention deficit hyperactivity disorder (ADHD). Psychopharmacology 202: 531-539.

Dickstein SG, Bannon K, Castellanos FX, Milham MP (2006). The neural correlates of attention deficit hyperactivity disorder: an ALE meta-analysis. J Child Psychol Psychiatry 47: 1051-1062.

Durston S, Tottenham NT, Thomas KM, Davidson MC, Eigsti IM, Yang YH et al (2003). Differential patterns of striatal activation in young children with and without ADHD. Biol Psychiatry 53: 871-878.

Eshleman AJ, Carmolli M, Cumbay M, Martens CR, Neve KA, Janowsky A (1999). Characteristics of drug interactions with recombinant biogenic amine transporters expressed in the same cell type. J Pharmacol Exp Ther 289: 877-885.

Gatley SJ, Pan D, Chen R, Chaturvedi G, Ding YS (1996). Affinities of methylphenidate derivatives for dopamine, norepinephrine and serotonin transporters. Life Sci 58: 231-239.

Gershon J (2002). A meta-analytic review of gender differences in ADHD. J Atten Disord 5: 143-154.

Goldberg D, Murray R (2002). Maudsley Handbook of Practical Psychiatry. Oxford University Press: Oxford.

Goodman R, Scott S (1999). Comparing the strengths and difficulties questionnaire and the child behavior checklist: Is small beautiful? J Abn Child Psychol 27: 17-24.

Hannestad J, Gallezot JD, Planeta-Wilson B, Lin SF, Williams WA, van Dyck CH et al (2010). Clinically relevant doses of methylphenidate significantly occupy norepinephrine transporters in humans in vivo. Biol Psychiatry 68: 854-860.

Konrad K, Eickhoff SB (2010). Is the ADHD brain wired differently? A review on structural and functional connectivity in attention deficit hyperactivity disorder. Hum Brain Mapp 31: 904-916.

Konrad K, Neufang S, Fink GR, Herpertz-Dahlmann B (2007). Long-term effects of methylphenidate on neural networks associated with executive attention in children with ADHD: results from a longitudinal functional MRI study. J Am Acad Child Adolesc Psychiatry 46: 1633-1641.

Konrad K, Neufang S, Hanisch C, Fink GR, Herpertz-Dahlmann B (2006). Dysfunctional attentional networks in children with attention deficit/hyperactivity disorder: evidence from an eventrelated functional magnetic resonance imaging study. Biol Psychiatry 59: 643-651.

Krause J (2008). SPECT and PET of the dopamine transporter in attention-deficit/hyperactivity disorder. Expert Rev Neurother 8: 611-625.

Langleben DD, Monterosso J, Elman I, Ash B, Krikorian G, Austin G (2006). Effect of methylphenidate on Stroop Color-Word task performance in children with attention deficit hyperactivity disorder. Psychiatry Res 141: 315-320.

Li CSR, Yan P, Chao HHA, Sinha R, Paliwal P, Constable RT et al (2008). Error-specific medial cortical and subcortical activity during the stop signal task: a functional magnetic resonance imaging study. Neuroscience 155: 1142-1151.

Mahone EM, Wodka EL. (2008). The neurobiological profile of girls with ADHD. Dev Disabil Res Rev 14: 276-284.

Matthews JNS, Altman DG. (1996). Statistics notes: interaction 2: compare effect sizes not $\mathrm{P}$ values. BMJ 313: 808. 
Merikangas KR, He JP, Brody D, Fisher PW, Bourdon K, Koretz DS (2010). Prevalence and treatment of mental disorders among US children in the 2001-2004 NHANES. Pediatrics 125: 75-81.

Montgomery AJ, Asselin MC, Farde L, Grasby PM (2007). Measurement of methylphenidate-induced change in extrastriatal dopamine concentration using [C-11]FLB 457 PET. J Cereb Blood Flow Metab 27: 369-377.

Moron JA, Brockington A, Wise RA, Rocha BA, Hope BT (2002). Dopamine uptake through the norepinephrine transporter in brain regions with low levels of the dopamine transporter: evidence from knock-out mouse lines. J Neurosci 22: 389-395.

Mullane JC, Corkum PV, Klein RM, McLaughlin E (2009). Interference control in children with and without ADHD: a systematic review of flanker and Simon task performance. Child Neuropsychol 15: 321-342.

Peterson BS, Potenza MN, Wang ZS, Zhu HT, Martin A, Marsh R et al (2009). An fMRI study of the effects of psychostimulants on default-mode processing during Stroop task performance in youths With ADHD. Am J Psychiatry 166: 1286-1294.

Pliszka SR, Glahn DC, Semrud-Clikeman M, Franklin C, Perez R, Xiong JJ (2006). Neuroimaging of inhibitory control areas in children with attention deficit hyperactivity disorder who were treatment naive or in long-term treatment. Am J Psychiatry 163: 1052-1060.

Raven J (1960). Guide to the Standard Progressive Matrices. HK Lewis: London.

Rubia K (2010). 'Cool' inferior fronto-striatal dysfunction in attention deficit hyperactivity disorder (ADHD) versus 'hot' ventromedial orbitofronto-limbic dysfunction in conduct disorder: a review. Biol Psychiatry (in press) doi:10.1016/j.biopsych.2010.09.023.

Rubia K, Cubillo A, Smith AB, Woolley J, Heyman I, Brammer MJ (2010a). Disorder-specific dysfunction in right inferior prefrontal cortex during two inhibition tasks in boys with attentiondeficit hyperactivity disorder compared to boys with obsessivecompulsive disorder. Hum Brain Mapp 31: 287-299.

Rubia K, Cubillo A, Woolley J, Brammer MJ, Smith AB (2011). Disorder-specific dysfunctions in patients with attention-deficit/ hyperactivity disorder compared to patients with obsessivecompulsive disorder during interference inhibition and attention allocation. Hum Brain Mapp (in press).

Rubia K, Halari R, Christakou A, Taylor E (2009a). Impulsiveness as a timing disturbance: neurocognitive abnormalities in attention-deficit hyperactivity disorder during temporal processes and normalization with methylphenidate. Philos Trans $R$ Soc Lond Ser B 364: 1919-1931.

Rubia K, Halari R, Cubillo A, Mohammad A, Scott S, Brammer M (2010b). Disorder-specific inferior frontal dysfunction in boys with pure Attention-Deficit/Hyperactivity Disorder compared to boys with pure CD during cognitive flexibility. Hum Brain Mapp 31: 1823-1833.

Rubia K, Halari R, Cubillo A, Mohammad M, Taylor E (2009b). Methylphenidate normalises activation and functional connectivity deficits in attention and motivation networks in medication-naïve children with ADHD during a Rewarded Continuous Performance Task. Neuropharmacology 57: 640-652.

Rubia K, Halari R, Smith AB, Mohammad M, Scott S, Brammer MJ (2009c). Shared and disorder-specific prefrontal abnormalities in boys with pure attention-deficit/hyperactivity disorder compared to boys with pure CD during interference inhibition and attention allocation. J Child Psychol Psychiatry 50: 669-678.

Rubia K, Halari R, Smith AB, Mohammed M, Scott S, Giampietro V et al (2008). Dissociated functional brain abnormalities of inhibition in boys with pure conduct disorder and in boys with pure attention deficit hyperactivity disorder. Am J Psychiatry 165: 889-897.

Rubia K, Overmeyer S, Taylor E, Brammer M, Williams SC, Simmons A et al (1999). Hypofrontality in attention deficit hyperactivity disorder during higher-order motor control: a study with functional MRI. Am J Psychiatry 156: 891-896.

Rubia K, Smith A, Brammer M, Taylor E (2007a). Performance of children with attention deficit hyperactivity disorder (ADHD) on a test battery for impulsiveness. Child Neuropsychol 30: 659-695.

Rubia K, Smith A, Halari R, Matukura F, Mohammad M, Taylor E et al (2009d). Disorder-specific dissociation of orbitofrontal dysfunction in boys with pure conduct disorder during reward and ventrolateral prefrontal dysfunction in boys with pure Attention-Deficit/Hyperactivity Disorder during sustained attention. Am J Psychiatry 166: 83-94.

Rubia K, Smith AB, Brammer MJ, Taylor E (2003). Right inferior prefrontal cortex mediates response inhibition while mesial prefrontal cortex is responsible for error detection. NeuroImage 20: $351-358$

Rubia K, Smith AB, Brammer MJ, Taylor E (2007b). Temporal lobe dysfunction in medication-naive boys with attention-deficit/ hyperactivity disorder during attention allocation and its relation to response variability. Biol Psychiatry 62: 999-1006.

Rubia K, Smith AB, Brammer MJ, Toone B, Taylor E (2005). Abnormal brain activation during inhibition and error detection in medication-naive adolescents with ADHD. Am J Psychiatry 162: 1067-1075.

Rubia K, Smith AB, Taylor E, Brammer M (2007c). Linear agecorrelated functional development of right inferior frontostriato-cerebellar networks during response inhibition and anterior cingulate during error-related processes. Hum Brain Mapp 28: 1163-1177.

Rubia K, Smith AB, Woolley J, Nosarti C, Heyman I, Taylor E et al (2006). Progressive increase of frontostriatal brain activation from childhood to adulthood during event-related tasks of cognitive control. Hum Brain Mapp 27: 973-993.

Schiffer WK, Volkow ND, Fowler JS, Alexoff DL, Logan J, Dewey SL (2006). Therapeutic doses of amphetamine or methylphenidate differentially increase synaptic and extracellular dopamine. Synapse 59: 243-251.

Schoenbaum G, Roesch MR, Stalnaker TA (2006). Orbitofrontal cortex, decision-making and drug addiction. Trends Neurosci 29: $116-124$

Shafritz KM, Marchione KE, Gore JC, Shaywitz SE, Shaywitz BA (2004). The effects of methylphenidate on neural systems of attention in attention deficit hyperactivity disorder. $\mathrm{Am} \mathrm{J}$ Psychiatry 161: 1990-1997.

Shaw P, Sharp WS, Morrison M, Eckstrand K, Greenstein DK, Clasen LS et al (2009). Psychostimulant treatment and the developing cortex in attention deficit hyperactivity disorder. Am J Psychiatry 166: 58-63.

Smith AB, Taylor E, Brammer M, Toone B, Rubia K (2006). Taskspecific hypoactivation in prefrontal and temporoparietal brain regions during motor inhibition and task switching in medication-naive children and adolescents with attention deficit hyperactivity disorder. Am J Psychiatry 163: 1044-1051.

Solanto M, Newcorn J, Vail L, Gilbert S, Ivanov I, Lara R (2009). Stimulant drug response in the predominantly inattentive and combined subtypes of attention-deficit/hyperactivity disorder. J Child Adolesc Psychopharmacol 19: 663-671.

Staller JA, Faraone SV (2007). Targeting the dopamine system in the treatment of attention-deficit/hyperactivity disorder. Expert Rev Neurother 7: 351-362.

Stevens MC, Pearlson GD, Kiehl KA (2007). An FMRI auditory oddball study of combined-subtype attention deficit hyperactivity disorder. Am J Psychiatry 164: 1737-1749.

Talairach J, Tournoux P (1988). Co-planar Stereotaxic Atlas of the Brain Thieme. New York.

Tamm L, Menon V, Reiss AL (2006). Parietal attentional system aberrations during target detection in adolescents with attention deficit hyperactivity disorder: event-related fMRI evidence. $A m \mathrm{~J}$ Psychiatry 163: 1033-1043. 
Tannock R, Schachar RJ, Carr RP, Chajczyk D, Logan GD (1989). Effects of methylphenidate on inhibitory control in hyperactivechildren. J Abn Child Psychol 17: 473-491.

Thirion B, Pinel P, Meriaux S, Roche A, Dehaene S, Poline JB (2007). Analysis of a large fMRI cohort: statistical and methodological issues for group analyses. NeuroImage 35: 105-120.

Vaidya CJ, Austin G, Kirkorian G, Ridlehuber HW, Desmond JE, Glover GH et al (1998). Selective effects of methylphenidate in attention deficit hyperactivity disorder: a functional magnetic resonance study. Proc Natl Acad Sci USA 95: 14494-14499.

Vaidya CJ, Bunge SA, Dudukovic NM, Zalecki CA (2005). Altered neural substrates of cognitive control in childhood ADHD: evidence from functional magnetic resonance imaging. Am J Psychiatry 162: 1605-1613.

Valera EM, Brown A, Biederman J, Faraone SV, Makris N, Monuteaux MC et al. (2010). Sex differences in the functional neuroanatomy of working memory in adults with ADHD. Am J Psychiatry 167: 87-94.

van Mourik R, Oosterlaan J, Sergeant JA (2005). The Stroop revisited: a meta-analysis of interference control in $\mathrm{AD} / \mathrm{HD}$. J Child Psychol Psychiatry 46: 150-165.

Volkow N, Ding Y-S, Fowler J, Wang G, Logan J, Gatly J et al (1995). Is methyphenidate like cocaine? Studies on their phamacokinetics and distribution in the human brain. Arch Gen Psychiatry 52: 456-463.

Volkow N, Wang G, Fowler J, Logan J, Angrist B, Hitzemann R et al (1997). Effects of methylphenidate on regional brain glucose metabolism in humans: relationship to dopamine D2 receptors. Am J Psychiatry 154: 50-55.

Volkow ND, Fowler JS, Wang G, Ding Y, Gatley SJ (2002a). Mechanism of action of methylphenidate: insights from PET imaging studies. J Atten Disord 6(Suppl 1): S31-S43.

Volkow ND, Fowler JS, Wang GJ, Ding YS, Gatley SJ (2002b). Role of dopamine in the therapeutic and reinforcing effects of methylphenidate in humans: results from imaging studies. Eur Neuropsychopharmacol 12: 557-566.

Volkow ND, Wang GJ, Fowler JS, Gatley SJ, Logan J, Ding YS et al (1998). Dopamine transporter occupancy by intravenous methylphenidate: implications for its reinforcing effects. Soc Neurosci Abstr 24: 778.

Volkow ND, Wang GJ, Ma YM, Fowler JS, Wong C, Jayne M et al (2006). Effects of expectation on the brain metabolic responses to methylphenidate and to its placebo in non-drug abusing subjects. NeuroImage 32: 1782-1792.

Volkow ND, Wang GJ, Newcorn J, Fowler JS, Telang F, Solanto MV et al (2007a). Brain dopamine transporter levels in treatment and drug naive adults with ADHD. NeuroImage 34: 1182-1190.

Volkow ND, Wang GJ, Newcorn J, Telang F, Solanto MV, Fowler JS et al (2007b). Depressed dopamine activity in caudate and preliminary evidence of limbic involvement in adults with attention-deficit/hyperactivity disorder. Arch Gen Psychiatry 64: 932-940.

Wilens TE (2008). Effects of methylphenidate on the catecholaminergic system in attention-deficit/hyperactivity disorder. J Clin Psychopharmacol 28(Suppl 2): S46-S53.

Willcutt EG, Doyle AE, Nigg JT, Faraone SV, Pennington BF (2005). Validity of the executive function theory of attentiondeficit/hyperactivity disorder: a meta-analytic review. Biol Psychiatry 57: 1336-1346.

This work is licensed under the Creative Commons Attribution-NonCommercial-No Derivative Works 3.0 Unported License. To view a copy of this license, visit http://creativecommons.org/ licenses/by-nc-nd/3.0/

Supplementary Information accompanies the paper on the Neuropsychopharmacology website (http://www.nature.com/npp) 